\title{
Video Article \\ Visualizing Efficacy of Pesticides Against Disease Vector Mosquitoes in the Field
}

\author{
Seth C. Britch ${ }^{1}$, Kenneth J. Linthicum ${ }^{1}$, Robert L. Aldridge ${ }^{1}$, Frances V. Golden ${ }^{1}$, Todd W. Walker ${ }^{2}$ \\ ${ }^{1}$ Mosquito \& Fly Research Unit, United States Department of Agriculture, Agricultural Research Service Center for Medical, Agricultural, \& Veterinary Entomology \\ ${ }^{2}$ East Baton Rouge Mosquito Abatement and Rodent Control
}

Correspondence to: Seth C. Britch at Seth.Britch@ars.usda.gov

URL: https://www.jove.com/video/58440

DOI: doi: $10.3791 / 58440$

Keywords: Environmental Sciences, Issue 145, Public health pesticides, vector control, ecological regions, peridomestic, sylvatic, military operational entomology, adulticide, larvicide, Mobile Pesticide App, Deployed War Fighter Protection (DWFP) Research Program, geographic information system (GIS)

Date Published: 3/16/2019

Citation: Britch, S.C., Linthicum, K.J., Aldridge, R.L., Golden, F.V., Walker, T.W. Visualizing Efficacy of Pesticides Against Disease Vector Mosquitoes in the Field. J. Vis. Exp. (145), e58440, doi:10.3791/58440 (2019).

\section{Abstract}

Efficacy of public health pesticides targeting nuisance and disease-vector insects such as mosquitoes, sand flies, and filth-breeding flies is not uniform across ecological zones. To best protect public and veterinary health from these insects, the environmental limitations of pesticides need to be investigated to inform effective use of the most appropriate pesticide formulations and techniques. We have developed a research program to evaluate combinations of pesticides, pesticide application equipment, and application techniques in hot-arid desert, hot-humid tropical, warm and cool temperate, and urban locations to derive pesticide use guidelines specific to target insect and environment. To these ends we designed a system of protocols to support efficient, cost-effective, portable, and standardized evaluation of a diverse range of pesticides and equipment across multiple environments. At the core of these protocols is the use of an array of small cages with colony-reared sentinel mosquitoes (adults and immatures) and sand flies (adults), strategically arranged in natural habitats and exposed to pesticide spray. Spatial and temporal patterns of pesticide efficacy are derived from percent mortality in sentinel cages, then mapped and visualized in a geographic information system. Maps of sentinel mortality data may be statistically compared to evaluate relative efficacy of a pesticide across multiple environments, or to study multiple pesticides in a single environment. Protocols may be modified to accommodate a variety of scenarios, including, for example, the vertical orientation of sentinels in canopy habitats or simultaneous testing of ground and aerial application methods.

\section{Video Link}

The video component of this article can be found at https://www.jove.com/video/58440/

\section{Introduction}

Efficacy of public health pesticides targeting nuisance and disease-vector insects such as mosquitoes, sand flies, and filth-breeding flies is not uniform across desert, tropical, temperate, or urban ecological zones ${ }^{1}$. Certain key species in these three groups of insects are important vectors of parasites, viruses, filarial worms, and bacteria that cause significant diseases in humans, pets, and livestock worldwide. To best protect public and veterinary health, the environmental limitations of pesticides must be investigated to inform effective use of the most appropriate pesticide formulations and techniques. Public health pesticide manufacturers are not required by the U.S. Environmental Protection Agency to specify the expected efficacy of a formulation across a range of environments or target insects, yet these pesticides are used for mosquito and vector control across multiple ecological zones in the U.S. and around the world.

We have developed a research program to evaluate numerous combinations of pesticides and pesticide application equipment and techniques in hot-arid desert, hot-humid tropical, warm and cool temperate, and urban locations, in order to derive pesticide use guidelines specific to the target insect and environment ${ }^{1}$. In this program, we evaluate pesticides that target adult stages of mosquitoes and sand flies (adulticides) and immature stages of mosquitoes (larvicides) using pesticide application equipment that is hand carried, truck- or aircraft-mounted, and installed in fixed locations. Then, four major outdoor pesticide application techniques are evaluated: (1) ultra-low volume (ULV) or thermal fog aerosol space sprays of adulticides designed for rapid knockdown of target insects, (2) a variant of the first technique in which liquid larvicides are applied with ULV or thermal fog for short- or long-term suppression of immature stages of target insects, (3) timed misting sprays from fixed locations designed to repel or kill, and (4) low volume (LV) cold mist sprays of residual pesticides designed to apply long-lasting toxic or repellent coatings on a variety of natural or artificial substrates. Presented here are the detailed methods for conducting techniques (1) and (2) mentioned above. Methods for (3) will be presented in separate studies, and techniques in (4) are described in brief in earlier publications ${ }^{2,3,4}$.

To accomplish this complex research program, we designed a system of protocols to support efficient, cost-effective, portable, and repeatable/ standardized evaluation of aerosol adulticide and larvicide techniques with diverse pesticides/equipment combinations across multiple environments. At the core of these protocols is the use of colony-reared sentinel mosquitoes (adults and immatures) and sand flies (adults) to indicate spatial and temporal patterns of pesticide efficacy. In the case of adulticide applications, sentinel adult mosquitoes or sand flies are contained in small single-use disposable cages distributed in structured arrays through the target area and an untreated control area. For 
larvicide applications, small single-use disposable cups are similarly distributed to collect sprayed larvicide droplets for later introduction of water and sentinel colony-reared immature mosquitoes. Next, we record percent mortality in sentinel cages, or percent adult development in sentinel cups, at set intervals post-spray and use these data to produce electronic maps of spatial and temporal efficacy in a geographic information system (GIS) that may be quantitatively compared between and among environments.

Using sentinel cages of colony reared insects to evaluate pesticide efficacy in the field is a long-established practice ${ }^{5,6}$, and using empty plastic sentinel cups for collecting sprayed larvicide is emerging in the literature ${ }^{7}$. However, our electronic mapping of efficacy to visualize spatial and temporal patterns of mortality is an innovation that greatly improves investigation of mortality otherwise presented in flat table formats. Also, the high throughput cage loading system and the modular cage deployment system adaptable to diverse scenarios described here are unique to our program. Other research programs approach evaluation of pesticide applications in the field differently. Current popular methods include capturing and analyzing dye-labeled pesticide droplets from sprays in the field on spinning glass slides ${ }^{8}$ or acrylic rods ${ }^{9}$, which is a longestablished process producing data that can be electronically mapped and visualized.

One drawback is that droplet size and density measurements from collection media are only estimated from a small proportion of the total collection surface, with software-assisted microscopy of fields of view that are unfortunately highly subjective. Also, maps of droplet distribution and density do not fully illustrate pesticide efficacy, because the assumption is that the presence of a threshold number of droplets of a certain size automatically indicates target insect mortality. This assumption does not account for mortality from evaporative products from droplets through the target area that may also induce mortality ${ }^{10}$, or that a lower number of droplets or other droplet sizes may kill some proportion of target individuals. The original rationale ${ }^{10,11,12}$ is that an aerosol pesticide is designed to impinge small droplets on actively flying target insects. However, our observations in the field, including reductions in natural populations after spraying when target insects are not actively flying, suggest that droplets or evaporative products from droplets are reaching targets that are not flying but rather hidden in resting refugia (unpublished data 2011). Also, we have observed in analysis of a field spray application (via simultaneous droplet capture, pesticide active ingredient capture, and sentinel cages) that maps of droplet distribution, active ingredient distribution, and mortality are not concordant (unpublished data 2010).

Another popular approach to evaluating pesticide efficacy is deploying sentinel cages in a grid delineated in a flat mowed homogeneous field with no obstructions to the pesticide plume, and under near ideal meteorological conditions (e.g., consistent winds $<10 \mathrm{mph}$ and wind direction perpendicular to the spray line). Still, others approach this by measuring efficacy with sentinels placed in wind tunnels ${ }^{14}$. These approaches provide one perspective on pesticide efficacy, but are less likely to realize operational efficacy under non-ideal field conditions (heterogeneous habitats that include obstructions to pesticide flow and variable if not under sub-optimal meteorological conditions). Seeking evidence in support of absolute efficacy is not realistic. Operational conditions are rarely ideal, and choosing formulations based on tests in wind tunnels or direct application in engineered parks may be misleading.

In our research, we use natural field sites and the given meteorological conditions (but not in rain or extreme winds that are outside the limits of any operational program). This is likely more informative for operational vector control when observing reasonable efficacy in a pesticide formulation despite poor environmental conditions, heterogeneous habitat, and obstructions to pesticide flow. Whenever possible, it is recommended to supplement sentinel insect mortality data with before- and after-surveillance of natural target insect populations in the treatment and control areas, as a bridge between controlled exposure and realized exposure to the focal pesticide. However, surveillance of natural populations is not sufficient to determine whether pesticide application produces mortality in a target population or whether target insects actually move from the target area after detecting oncoming pesticide aerosols.

Regardless of the caveats to any evaluation of pesticide sprays in the field, electronic mapping of mortality data in a GIS (as opposed to flat presentation of mortality data in tables) retains the quantitative attributes capable of rigorous comparison across trials and also provides a means for quick, visual assessment. With data captured in the GIS, researchers can set thresholds for pesticide efficacy and visualize the relative capability of a focal pesticide across multiple environments, or they can compare capabilities of multiple pesticides within a single environment across a variety of application equipment and techniques.

\section{Protocol}

Note: This protocol is written specifically for field trials targeting adult mosquitoes. Information on modifications necessary for immature mosquitoes, other adult sentinel insect species, and unique scenarios is included in the discussion.

\section{Sentinel Insect Rearing and Sentinel Cage Preparations}

1. Constructing adult sentinel cages

1. Determine the total number of sentinel cages needed for the field trial by including treatment and control areas and the number of replicates planned, then add 5\% to offset losses due to damage or unusual mortality (see step 2.1). Prepare $5 \times 5$ in tulle mesh squares ( 2 per sentinel cage) using a rolling disk cutter, straight edge, and cutting board with bulk tulle yardage. Store the tulle mesh squares in batches of 50 in 1 qt resealable plastic bags.

2. Prepare the sentinel cages by carefully pushing the flat cardboard disks out of the bottoms of new $1 / 2$ pt cardboard ice cream cups to create open-ended $2 \times 3$ in cylinders. For each cup, remove the cardboard disks out of 2 lids to create open-ended bezels ( 1 for each end of the cylinder). Secure one tulle mesh square to one end of the cylinder by trapping it under the bezel as the bezel becomes tightly fitted to the cylinder.

3. For each sentinel cage, fasten a hook-and-loop cable tie to a rubber band, and secure the rubber band around the bezel that is in place. Bag the sentinel cages in batches of 50 , with a separate bag containing 50 bezels and a bag of 50 tulle mesh squares. Note: Tulle mesh size should be the minimum gap size to contain the particular sentinel species to be placed in the cage. For example, Aedes aegypti mosquitoes will require a smaller mesh size than Culex quinquefasciatus mosquitoes. 


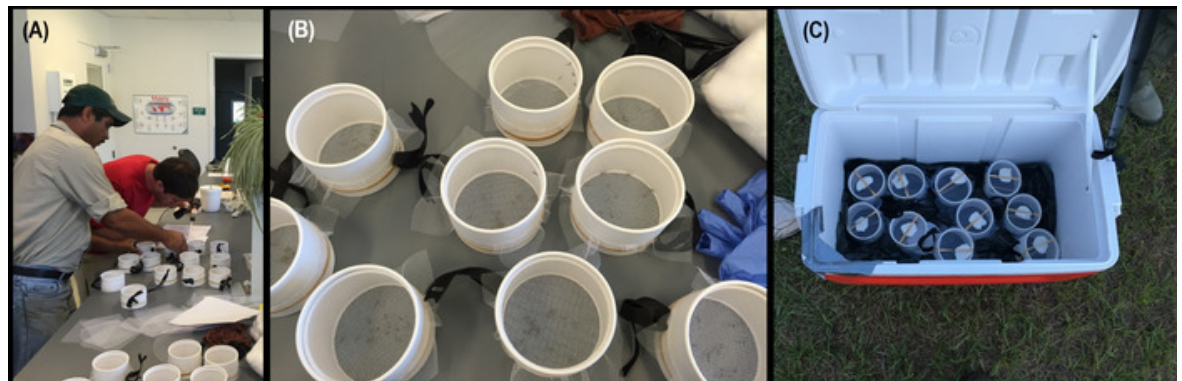

Figure 1: Sentinel cage preparation. (A) Shown here are two personnel loading sentinel cages with anaesthetized mosquitoes spread on a large white sheet of paper. The stack of tulle mesh squares in the foreground ready for placement on the waiting open sentinel cages should be noted. (B) Shown are several loaded sentinel cages, awaiting placement of $10 \%$ sucrose cotton balls and realignment of the rubber band. (C) Shown are sentinel cages in a cooler, ready for deployment in the field. Please click here to view a larger version of this figure.

2. Scheduling and rearing colony insects

1. For sentinel insects, select a species that is most appropriate for the environment, season, and medical threat in the location where the trial will take place. Consider the colony capabilities and development time to adult for that species; for instance, consider if the colony will be able to provide the number of specimens needed for the number of sentinel cages required in the time frame.

2. For all rearing, ensure population rearing cages are kept in a controlled environment and are large enough for the number of adults planned, and that a feeding and cleaning regime is established and carefully followed to reduce later stress-related mortality that will confound field efficacy trials. For extensive field trials, plan to rear sequential batches in separate well-labeled population cages.

3. Use only female mosquitoes in sentinel cages, preferably 3-5 d post-eclosion. Mosquito pupae can be sexed under a dissecting scope or physically separated using a glass pupal separator to reduce sorting effort with adults at the sentinel cage loading step.

Note: A colony must be able to supply double the number of mosquitoes planned for use in sentinel cages, because only females are used for sentinels. Use 10 adult females for each sentinel cage, as this number is quick to load, quick to count for mortality, and easy to determine whether any have escaped. It is essential to back-plan from the proposed spray date so that 3-to 5-day-old adult mosquitoes are available. However, the $3-5 \mathrm{~d}$ spread does provide some margin in case a weather hold prevents spraying.

3. Loading sentinel cages

1. Prepare a $\mathrm{CO}_{2}$ chamber by lining the bottom of a new $1 / 2$ gal plastic food storage container with cotton balls. Direct one end of a 6 -ft length of polyurethane tubing $(1 / 4$ inch diameter $)$ into the cotton balls through a tight-fitting hole punched low in one side of the container. Attach a $20 \mathrm{lb} \mathrm{CO} 2$ bottle with a regulator to the other end of the tube and place the lid loosely on the container to allow gas to escape after diffusing through the cotton balls and circulating through the container. Tape the tube to the table top to prevent the lightweight chamber from being pulled or knocked to the floor. Set a flow rate appropriate to the species being used (start at $\sim 7 \mathrm{~L} / \mathrm{min}$ and make adjustments as needed).

2. Prepare $10 \%$ sucrose solution in a new $1 / 2$ gal plastic food container and submerge 100 cotton balls so they become fully saturated. Lay out the sentinel cages from step 1.1 in batches of 25 or 50 as space permits, with the tulle mesh squares and loose bezels on hand nearby.

3. Remove mosquitoes from the population cage with a mechanical aspirator capable of channeling mosquitoes directly into removable ventilated collection tubes. No more than $\sim 10$ mosquitoes should be aspirated into each tube, and no more tubes than can be processed within $10 \mathrm{~min}$ should be filled to mitigate stress. Place 2-4 tubes at a time into the $\mathrm{CO}_{2}$ chamber and set a timer for a 4-min exposure to anaesthetize the mosquitoes for subsequent sexing and transfer to sentinel cages.

4. Once the timer chimes, remove all tubes from $\mathrm{CO}_{2}$ chamber and carefully shake out anaesthetized mosquitoes from the tubes to scatter them evenly across a clean white $11.5 \times 17$-in sheet of paper. Set the timer for $4 \mathrm{~min}$, which is the approximate time before mosquitoes will become active again. Use a pistol aspirator ${ }^{15,16}$ or soft-touch insect forceps to pick out batches of 20 adult females and immediately place them in the waiting open sentinel cages. Once all females have been picked out of the anaesthetized batch, use a separate mechanical aspirator to collect all remaining male mosquitoes which will be destroyed by freezing.

5. As each waiting open sentinel cage receives a batch of 20 adult female mosquitoes, trap a tulle mesh square with a bezel over the open upper end of the cage cylinder, and push down to complete the sentinel cage assembly. Once the tulle is in place, pivot the rubber band so that it lays across both mesh ends of the sentinel cage, with the hook-and-loop cable tie positioned against the side of the cylinder. Squeeze the majority of $10 \%$ sucrose solution out of a cotton ball so that it is still moist, just before the point of freely dripping, and gently tuck the cotton ball under the rubber band against the tulle mesh on one side of the sentinel cage, easily accessible to the mosquitoes.

6. Repeat steps 1.3.3-1.3.5 until all the sentinel cages are loaded.

Note: The cage loading procedure should include a minimum of two personnel: one to conduct all mosquito handling steps and another to conduct all cage handling steps.

4. Loading sentinel cage coolers

1. Prepare a 48-qt insulated picnic cooler for every $45-50$ sentinel cages (depending on the make/size of cooler) by placing a wet $18 \times 24-$ in cotton towel at the bottom covered by a 13-gal tall kitchen trash bag. The wet towel should be wrung out to the point of saturation but not dripping. Fold and arrange the 13-gal bag to completely cover the bottom of the cooler and at least 3 in from the bottom on all sides.

2. Load the cooler by carefully placing sentinel cages in stacked layers with the cotton balls facing up. Depending on the make of the cooler, there should be about 10-12 cages per layer and 4-5 layers. If possible, slightly offset each layer so that a cage does not completely cover the cage below to promote air flow. Cover the top layer with a second 13-gal trash bag (folded like the bottom trash bag) and spread a second wet towel on top of this bag.

3. Use duct tape to fasten a folded piece of cardboard in a front corner of the upper lip of the cooler, such that the lid is propped open about $3 / 8$ in to promote air exchange and prevent overheating. In each cooler include a 1-qt resealable plastic bag with 10 spare 
cotton balls soaked in $10 \%$ sucrose solution, and a separate bag with 5 spare rubber bands and 5 hook-and-loop cable ties (these components are occasionally lost from sentinel cages during deployment in the field). Throughout the time in the coolers in the lab and field, top off the sucrose solution in all cotton balls on cages as needed, using a dropper and $10 \%$ sucrose solution stored in a rugged container.

Note: With this method, typical colony mosquitoes may be loaded into sentinel cages and stored in coolers $24-28 \mathrm{~h}$ before use in field trials.

\section{Transporting sentinel cage coolers to the field}

1. During transport to field site keep sentinel cage coolers in a vehicle with air conditioning but protected from direct sunlight. At field sites in mild to warm temperate environments, the coolers can be stacked outdoors to acclimatize or kept in vehicles with air conditioning running; but in both cases, keep coolers out of direct sunlight. Watch for any ambient conditions outdoors that could affect sentinels in the coolers (e.g., vehicle exhaust, trash fires, etc.)

2. In extreme hot environments, keep coolers in vehicles with air conditioning running out of direct sunlight, but acclimatize the sentinels gradually to outdoor conditions before deployment to the treatment area to avoid death from shock. In all environments, consider testing one or two coolers of sentinel cages to determine how long they can be left out or how quickly they can be acclimatized to local outdoor conditions.

\section{Preparation of the Field Site}

1. Establishing the sentinel grid and control area

1. Select a study area that provides sufficient space for dispersal of the planned mode of pesticide application (aerial, truck, or portable) with a clear path for movement of the spray device, and clear of human settlement or activity. If possible, include a variety of vegetation density and general heterogeneity of the habitat to challenge spray penetration.

2. Design a grid of locations for sentinel cages that captures variations in distance from the sprayer, distance along the spray line, vegetation/obstacles to spray penetration, and height above the ground. Use a $300 \mathrm{ft}$ measuring tape reel to mark convenient intervals from the sprayer and along the spray line; for example, use multiples of $25 \mathrm{ft}$ intervals.

3. Produce a map of the grid by marking up a printout of a satellite image with a clear numbering code for sentinel cage locations and distribute it to the field team. Whenever possible, obtain GPS coordinates for each cage location or at least coordinates of prominent features that can later be used for registration points to build a spatially coherent map in the GIS.

4. Mark sentinel cage grid locations on the ground with flagging tape, glow sticks, and/or pin flags. Pesticide sprays are often conducted at dusk or night: attempt to clear distinct walking paths to all sentinel locations, including extra flagging tape and/or glow sticks to mark trip hazards or other significant obstacles.

5. Install sentinel cage mounting poles into the ground according to the grid design, and clearly label each pole with a code for its unique position with a permanent marker. At least 48 inches of the pole should be above ground. If the habitat does not naturally provide vegetation/obstacle challenges to pesticide spray dispersion, consider placing a $1 \mathrm{ft}^{3}$ cardboard box on its side with the top open next to each sentinel cage pole, to provide an additional sentinel location within simulated refugia. Air flow through the box can be adjusted by drilling $1 / 2$-in holes in one or more sides, and boxes should be labeled with a unique position code with a permanent marker.

6. If there is a risk of ants attacking sentinel mosquitoes, install sentinel cage mounting poles through a tight-fitting hole drilled in the center of an $18 \times 18$-in adhesive floor tile, with the sticky side facing upwards to trap ants before they can reach sentinels. The $1 \mathrm{ft}^{3}$ boxes from step 2.1 .5 can also be placed on the sticky side of such tiles to impede ants, and also to keep boxes in place in windy conditions.

7. Establish an upwind control area (consisting of a similar habitat and meteorology as the treatment area) for the deployment of untreated control sentinel cages exposed to the environment on poles and/or in $1 \mathrm{ft}^{3}$ boxes for the same amount of time as sentinels in the treatment area. A specific team of personnel with separate coolers of sentinel cages should be dedicated to handling all control sentinels.

8. Resources permitting, design a distribution of appropriate traps throughout treatment area, areas adjacent to treatment area, and control areas to conduct surveillance of local natural populations of mosquitoes that are closest to the species selected in 1.2.1. Begin daytime or overnight surveillance, depending on the species, during one or more of the 12-24 $\mathrm{h}$ periods before the spray trials are to take place. Continue surveillance throughout the spray trials, followed by surveillance for one or more periods after the spray trials. 


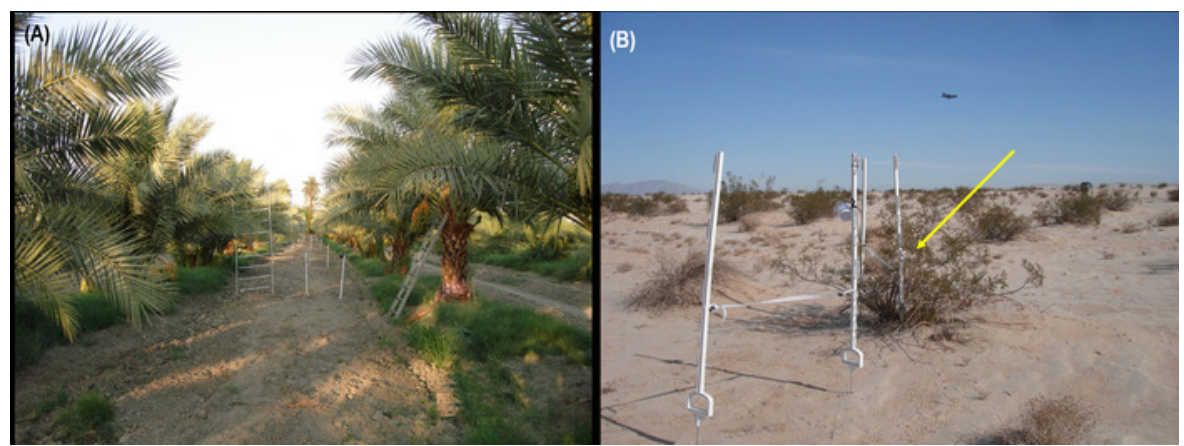

Figure 2: Prepare field site with sentinel cage poles. Two scenarios of sentinel cage poles distributed in (A) irrigated tropical microhabitat in a hot-arid region and (B) hot-arid desert. In (A), three kinds of sentinel apparatus are shown: the "ladder" on the left is a series of cotton ribbons suspended between PVC pipe to capture pesticide at various heights above ground; the pole in the middle is for sentinel mosquito cages; and the pole on the right supports a slide spinner to capture pesticide droplets. In (B) there is a similar cotton ribbon to capture pesticide, but it should be noted that the right-hand support of the apparatus in the foreground in the open doubles as a sentinel cage pole (the sentinel cage attached about one-third of the way down); in contrast, the apparatus in the background is placed so that the ribbon and the sentinel cage (indicated with yellow arrow) are sheltered within vegetation. The aircraft in the background is conducting a ULV pesticide spray. Please click here to view a larger version of this figure.

\section{Practicing safety}

1. Establish a communication system among the spray team, treatment area sentinel handling team, control area sentinel handling team, etc., via mobile phones (voice/text) or handheld radios.

2. Supply personal protective equipment (PPE) to all personnel that are appropriate for their roles and appropriate for the pesticide formulation and application equipment.

3. Establish a pesticide storage and mixing/loading area protected with secondary containment and stocked with emergency equipment, such as shovels and/or fire extinguishers for thermal fog applications, towels to contain spills or drips, waste containers, clean water, first aid supplies, and portable eyewash stations. Importantly, establish protocols in the cases of spills or exposure of personnel to chemicals.

3. Marking the spray line and recording meteorology

1. For driving or hand-carry applications, make sure the spray line is clear and that obstacles are identified and clearly marked with flagging tape, pin flags, and/or glow sticks. For aerial applications, make all appropriate arrangements with air traffic control.

2. The basic length of the spray line should match the dimensions of the grid; however, set the start and stop points well-before and wellafter the grid. Add approximately $10 \%$ of the basic length of the spray line to the beginning and end to increase the opportunity for the entire grid to be exposed, given inevitable shifts and imperfections in wind patterns (see representative results).

3. Whenever possible, anticipate major wind shifts, set up alternate spray line(s), and design the sentinel grid to be informative from both spray directions. The spray operator should be prepared to stop spraying briefly if the wind starts to gust badly or suddenly shifts, which can create a safety hazard. The operator can resume spraying when appropriate, and the trial can still produce usable efficacy data as long as controls are kept in the environment for a similar extended period.

4. Place at least one weather station with a data recorder capable of measuring temperature, relative humidity, wind speed, and wind direction in the spray area and another in the control area. Also, set up a simple wind indicator ribbon (about 2-3 ft of bright colored flagging tape) on a spare sentinel cage pole in view of the spray operator and field team to increase overall awareness of conditions. For aerial applications, the aircraft should ideally be capable of reporting meteorology at release height.

Note: Collaborate with a university, public health organization, mosquito and vector control district, or other agency with knowledge of the area proposed to conduct field trials, such that existing partnerships and understandings with landowners or land stewards may be built upon and help to develop the research program. Existing partnerships translate to site security, long-term use, and in some cases, critical buy-in and acceptance by the public. With structured grids, it is straightforward for local collaborators to develop their own numbering/labeling schemes for rapid and repeatable deployment, collection, and mortality checks of sentinel cages. Once the grid, spray line, and meteorology are set up, the materials can be left in place (for example, overnight until a convenient spray time or if there is a weather hold).

CAUTION: Cardboard boxes can deform when wet if it rains, and strong winds can move the heavy adhesive floor tiles.

\section{Deploying the Sentinel Cages}

1. Determine in advance the positioning of sentinel cages at each sentinel point in the grid, such as the height of cage on the mounting pole (including multi-height, where one might be placed at the bottom of the pole and another at the top), or whether cages will be placed in the 1 $\mathrm{ft}^{3}$ boxes.

2. Assign 2-person sentinel cage deployment teams: one to hold the cooler and another to pull out sentinel cages as needed. Alternatively, assign individuals to carry and place multiple cages depending on the environment and size of the grid. Always include extra sentinel cages in case one is dropped or stepped on, tulle is torn, or one is lost while moving through the grid.

3. Per the positioning regime as described in step 3.1, use the hook-and-loop cable tie to affix sentinel cages to poles and make sure cotton balls with $10 \%$ sucrose solution are still in place under the rubber band. Place cages on their sides in the $1 \mathrm{ft}^{3}$ boxes so that both tulle mesh ends are perpendicular to the ground. Orientation of cages on poles may be specified in advance; for instance, such that mesh tulle sides are in line with the planned spray direction and anticipated wind direction. In hot desert environments, angle the cages on the poles such that the insects are not in direct sunlight and may move into the shade of the side of the cage cylinder. 
4. In both treatment and control areas, clearly label each sentinel cage with a permanent marker as it is put in place with two pieces of information: (1) the pole and/or box unique position code for later mapping in the GIS, and (2) pre-spray mortality for later correction for spray-induced mortality or mortality from exposure to the environment regardless of spray. For example, if pre-spray mortality is zero, write "pre-0"; if 2 specimens are dead, write "pre-2", etc.

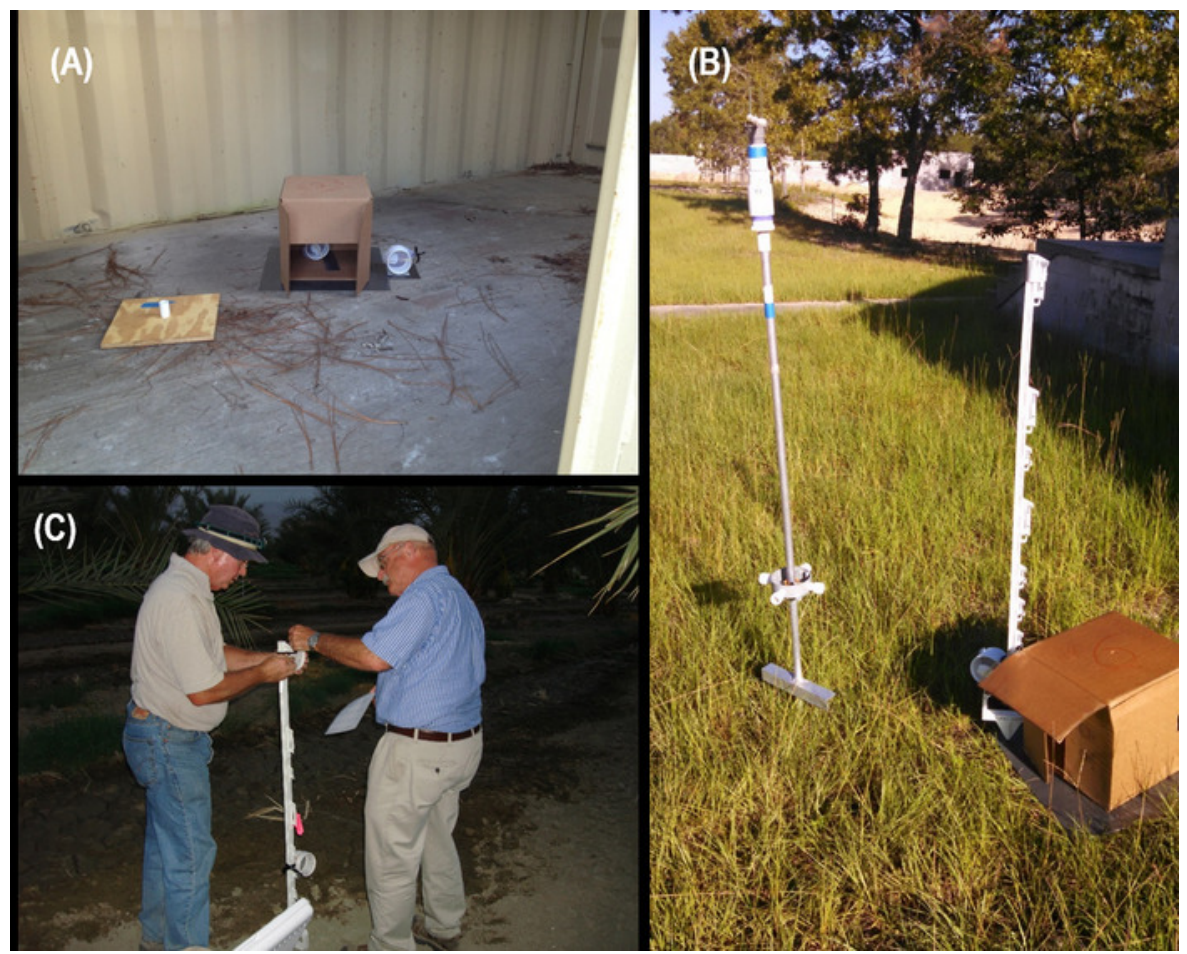

Figure 3: Placement of sentinel cages indoors and outdoors. Examples of pole placements and $1 \mathrm{ft}^{3}$ boxes $(\mathrm{A})$ indoors in a simulated rural residence, $(B)$ outdoors in a simulated neighborhood, and $(C)$ in a date palm grove in a hot-arid zone. Also shown are the sticky tiles under the box and under the sentinel cage placed in the open in (A), and the sentinel cage pole pin pushed through the sticky tile next to the box in (B) to reduce incursion of ants into sentinel cages. The plywood square with upright PVC mount to the left of the box in (A) is a support for a slide spinner. A similar apparatus may be used to support a sentinel cage pole to allow placement of sentinels at varying heights. Also shown is the slide spinner with slides on a mount to the left of the sentinel cage pole and box (B). In (C) is shown a high-low placement of two sentinel cages to investigate movement of the pesticide at different levels. The top of the opened sentinel cage cooler is just visible in the foreground. Please click here to view a larger version of this figure.

Note: Depending on size of the grid, ensure there are sufficient personnel to deploy all cages in an appropriate time frame to minimize environmental effects on sentinel mortality. It may be necessary to conduct practice runs with empty cages to determine whether enough personnel are present. It is understood that there will unavoidably be some spread in the exposure time of sentinel cages to the environment, owing to set-up time with the given number of cages per person. In very large sentinel grids (e.g., for some aerial applications), plan on coordinating multiple teams in several vehicles to place the sentinel cages within a reasonable time frame. It is inevitable that some mortality will occur across the sentinel cage mosquito population, so it must be accounted for with careful cage labeling prior to environmental exposure outside the coolers and prior to pesticide application.

\section{Conducting the Pesticide Application}

1. Assign a timekeeper to track application spray duration (start/stop times) and hold times using a stopwatch, clock, or digital video with time/ date stamps. The timekeeper can also be assigned a scribe to capture multiple key variables including the time at which sentinel cage deployment is completed, flying, driving, walking speed, application device flow rate, pesticide concentration and diluent, spray time, hold time, time at which cage collection is completed, and general habitat observations.

2. Prepare the pesticide application equipment per manufacturer guidelines and load the pesticide (and diluent if necessary), using the minimum amount to cover the target area plus a pre-determined amount to account for spray line volumes and pesticide reservoir sump to prevent cavitation during spray. Do not exceed application rates specified on the pesticide label and do not use pesticides not approved by the U.S. Environmental Protection Agency, unless an experimental use permit specific to the location of the field trial has been secured.

3. Establish a sufficient post-spray hold time that will allow pesticide spray plume to disperse throughout the sentinel cage grid before the sentinel cages are collected. Hold time is based on a balanced consideration of habitat/vegetation density, meteorological conditions (in particular extreme heat or wind), application device, application mode (aerial, ground vehicle, portable), target insect, and pesticide formulation, and may require preliminary trials to develop effectively.

4. Once sentinel cages are labeled and deployed in both treatment and control areas and all key personnel have communicated readiness, have the spray operator conduct a final check on wind speed and direction and initiate the aerial, ground, or portable sprayer pesticide application from the designated spray line or alternate spray line as needed. Set the flying, driving, or walking speeds depending on application device flow rate and pesticide label rate. 
5. Once the spray application is complete, have the timekeeper mark the beginning of the hold time and notify all personnel 1-2 min before the hold time will elapse, so that all personnel can move into position and prepare for collecting the sentinel cages.

CAUTION: Observe all safety precautions specified on the pesticide label in addition to standard pesticide applicator best practices. Pesticide applications should be conducted by a certified pesticide applicator with, if possible, additional certification in the public health pesticide category. Before the spray application ensure all personnel are upwind of application area, or in vehicles with air conditioning on recirculate if they must be in the target area, and/or are wearing appropriate PPE. Ensure all sentinel cage coolers are closed and that inside closed vehicles parked upwind of the spray application have the air conditioning on recirculate.

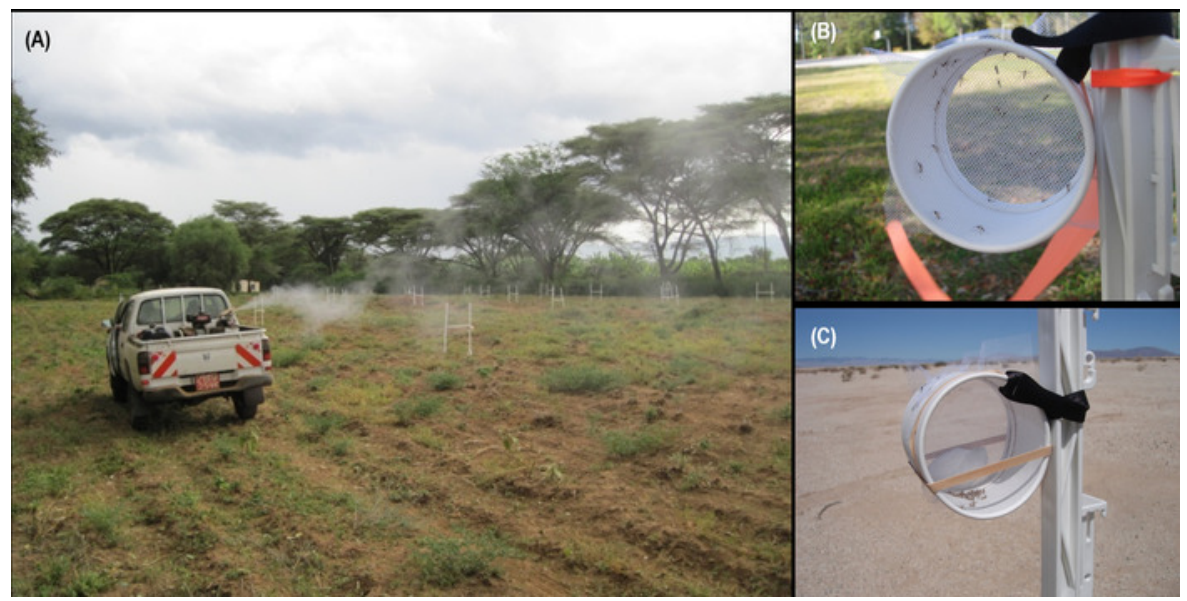

Figure 4: Vehicle mounted thermal fog spraying a grid in warm-arid equatorial location. A truck mounted ULV sprayer driving along a spray line directing pesticide spray through a grid of sentinel cages in an open field (A). Similar to Figure 2, each sentinel position is fitted with a second pole to support a cotton ribbon to collect pesticide droplets for later analysis with gas chromatograph/mass spectrometry. Close-ups of sentinel cages (B) in a warm temperate environment before spray and $(\mathrm{C})$ in a hot-arid environment after spray. Please click here to view a larger version of this figure.

\section{Collecting the Sentinel Cages and Recording Mortality Data}

1. Use the same sentinel cage teams to collect cages throughout the grid once the hold time has elapsed. All personnel handling postspray cages should wear disposable nitrile gloves to minimize human contact with pesticides. Return post-spray cages to the protective environment of the sentinel cage coolers as soon as possible, stored in a cool vehicle out of direct sunlight, and returned to the lab for processing as soon as possible.

2. In both treatment and control areas, it is critical to record post-spray mortality on each sentinel cage with a permanent marker as it is collected. Post-spray mortality count should include all dead mosquitoes in the cage, and this number should be written directly adjacent to the "pre-" count, but circled clearly enough to distinguish it from the pre-exposure mortality count. Later, the pre-count will be subtracted from the post-count to obtain the actual mortality from exposure to the pesticide (spray area) or environment (control area). Do not attempt to conduct this math in the field while hurrying to collect sentinel cages.

3. Attempt to return sentinel cages to the lab workspace within $6 \mathrm{~h}$ post-spray so that the $6 \mathrm{~h}$ mortality count can be recorded. In the lab, transfer all cages into plastic trays, discarding all rubber bands, hook-and-loop cables ties, and cotton balls. Each tray should hold approximately 20 sentinel cages stored with the mesh tulle parallel to the ground. Place a new $10 \%$ sucrose solution cotton ball on all cages and cover each tray with a new moist cotton towel, similar to those used in the sentinel cage coolers. Stack the trays on a lab bench or in an incubator using $1 / 2$-in PVC pipe spacers. Store the trays with cages from treatment areas well-separated or in different incubators that control cage trays.

4. At this point, transfer pre- and post-spray mortality data from each cage to data forms as the $6 \mathrm{~h}$ mortality count is conducted: one person wearing disposable nitrile gloves should take sentinel cages out of trays one-by-one for each cage, calling out the 1) unique location code, then the 2) pre-count and the immediate post-count read from the cage itself, followed by the 3) current $6 \mathrm{~h}$ count observed directly. A second person should record these data on pre-prepared forms having a row for each unique position code from the trial (Table 1). Repeat this process at $12 \mathrm{~h}$ and $24 \mathrm{~h}$ post-spray, storing the trays in stable conditions in between mortality counts.

5. Once the $24 \mathrm{~h}$ mortality count is complete, freeze all trays of the sentinel cages to kill all specimens, and then conduct final counts for each cage to obtain an accurate denominator for calculating percent mortality. If a specimen is missing from a cage, look directly below it in the tray, where it may have dried out and fallen through. Dispose of all sentinel cages, cotton towels, and plastic trash bag liners in sealed garbage bags in normal trash, and thoroughly clean all coolers and trays with soap and water and air-dry them in direct sunlight.

Note: Larval tray spacers are easily made from PVC stock pipe, with a notch cut in each end so they cannot roll off the trays. Spacers allow stacking of larval trays to save space in vehicles, incubators, or lab benches. For some field sites, it may not be possible to return to the lab within $6 \mathrm{~h}$; in which case, transfer cages from coolers to trays in the field using the above protocol and materials and stack them carefully in the air-conditioned vehicle. Recording mortality in each cage immediately after the post-spray hold time, and at approximately $6 \mathrm{~h}, 12 \mathrm{~h}$, and $24 \mathrm{~h}$ post-spray will provide information on knockdown (short-term) vs. long-term effects of the pesticide. 


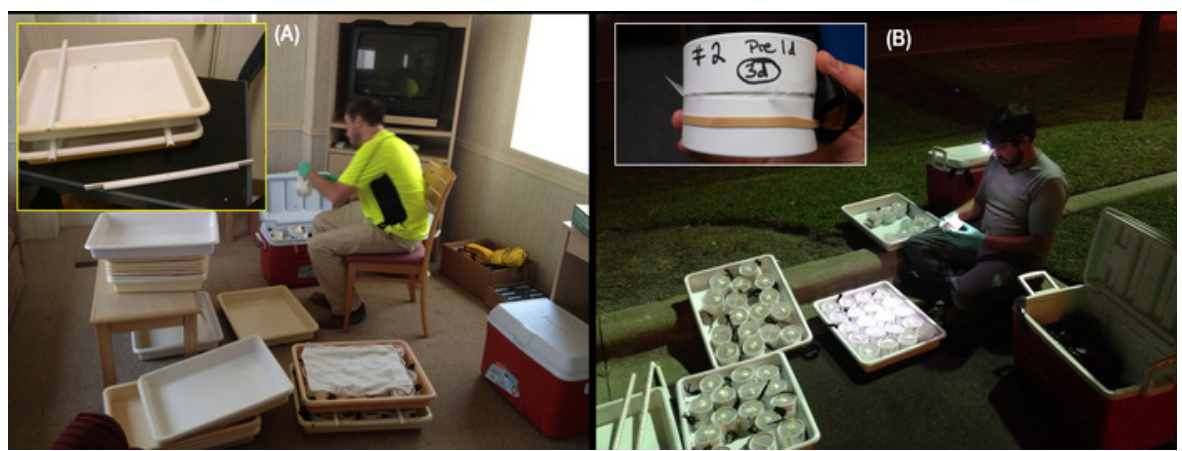

Figure 5: Processing sentinel cages post-spray. Two scenarios of transferring post-spray cages into stacked trays during the $6 \mathrm{~h}$ mortality check: (A) in a hotel room near a remote field site and (B) in a convenient parking lot returning to the lab from a distant field site. Note the PVC spacers in the inset photo in (A), moist towels covering cages laid out in trays in the main photo of $(A)$, and an example of a unique location code and pre- and circled post-spray mortality annotated directly on a sentinel cage in the inset photo in (B). Please click here to view a larger version of this figure.

\section{Processing, Analyzing, and Mapping Mortality Data}

1. Transfer all hand-recorded mortality data from the forms into an electronic spreadsheet and calculate 4 new columns for post-spray, $6 \mathrm{~h}, 12 \mathrm{~h}$, and $24 \mathrm{~h}$ mortality (all corrected for pre-spray mortality).

2. Correct all treatment area mortality data for control mortality in 4 additional new columns using the Abbott correction ${ }^{17}$ : calculate a mean control mortality across all control sentinels for each time period that mortality was recorded (i.e., post-spray, $6 \mathrm{~h}, 12 \mathrm{~h}$, and $24 \mathrm{~h}$; all corrected for pre-spray mortality), and use this mean to Abbott-correct the mortality in each treatment area sentinel cage for each corresponding time period. The threshold for acceptable control mortality may be arbitrarily determined based on the species of sentinel mosquito used but should not exceed $25 \%{ }^{18}$.

3. In a geographic information system map all unique sentinel cage locations using the GPS data gathered in step 2.1 .3 and create 4 columns for Abbott-corrected mortality for the 4 mortality time periods in the attribute table for these locations. Create an interpolated coverage for mortality for each of the 4 mortality time periods across all unique sentinel locations in the treatment area. Add these interpolated coverages to the map and use a stretched color ramp on a blue to red spectrum oriented low (blue) to high (red).

4. Enhance the map of the treatment area with an underlying satellite image and add sentinel cage positions (distinguishing treatment and control sentinels), spray line, driving direction, scale bar, north arrow, interpolated mortality color ramp key, key to symbols, and a detailed title with date, habitat type, pesticide and diluent used, application equipment used, and target species. Export a map image for each mortality time-period surface.

5. Add meteorological information as an inset graph and include a separate wind rose (polar plot) to show prevailing wind, wind speed, and variability throughout the spray period. Also, provide separate meteorological data for release height in the case of aerial applications.

6. Graph local natural population surveillance data as an index of the number of females of each species collected per trap per night. Output a series of graphs partitioned by location (adjacent to treatment area, inside treatment area, and inside control area) and period (sampled prior to, during, or following the spray trials) and place them separately as insets in the main GIS map to show effects on natural populations over time within, adjacent to, and away from the pesticide application.

\section{Representative Results}

Here are representative results presented from two unpublished field studies that included the core of the methods described above. In these studies, two aspects of adulticide efficacy against sentinel disease vector insects were investigated.

The first study (unpublished data 2010-2012) investigated whether diluent might influence efficacy of pesticide against mosquitoes in a hot-arid desert environment applied with a thermal fog device. We conducted three separate applications with a synergized permethrin adulticide capable of being diluted in either oil or water. Each of the applications was conducted using a truck-mounted thermal fog generator with a different diluent: either water, BVA13 mineral oil, or diesel. Then, a grid of at least 20 sentinel mosquito cages placed on poles in an open arid-land area (Figure 6) was used and meteorology was recorded using a portable weather recorder.

The second study (unpublished data 2011) investigated the relative efficacy of a single pesticide formulation applied simultaneously with two kinds of sprayers (ULV and thermal fog) in a hot equatorial environment against sand flies. We used two adjacent grids of 25 sentinel sand fly cages placed on poles in low grass-forb habitat of varying density in a large field in a hot equatorial valley basin (Figure 7). Meteorology was recorded using a portable weather recorder positioned between the two grids. One truck carried the ULV device and another carried the thermal fog generator, and both initiated sprays along the spray lines at the same time, each moving from east to west at a speed appropriate to the label-specified application rate and given flow rate of the sprayer.

In both field studies, after a 10-min hold time post-spray, we collected all sentinel cages from treatment and control areas, simultaneously initiating the process of recording post-spray mortality. Percent mortality data was then coded, corrected for observed background control sentinel mortality, and placed into a GIS coverage consisting of georeferenced points corresponding to the locations of the sentinel cages in the treatment area grid (Figure 6 and Figure 7). Despite the ideal situation in which a pesticide spray produces a 100\% kill throughout the target area, the realistic threshold for acceptable efficacy of a pesticide is arbitrary. Expectations of efficacy could vary with distance from the sprayer (e.g., setting a threshold of $95 \%$ mortality $50 \mathrm{ft}$ from the spray line, with $80 \%$ mortality at $250 \mathrm{ft}$ ). 
The results from both representative studies naturally show the spectrum of positive to negative outcomes, because the color ramp represents areas of 0 to $100 \%$ mortality (see color ramps in Figure 6 and Figure 7). All mortality data in the treatment area are normalized by the background mortality in the untreated control area to a threshold of $25 \%$ control mortality ${ }^{18}$, above which would have discarded due to excessive environmental or colony effects on mortality. The utility of the electronic mapping approach for visualizing mortality data is evident here: the researcher and (later) the reader can instantly understand the relative efficacy of the focal pesticides and diluents (Figure 6) or the focal pesticide sprayers (Figure 7) against mosquitoes or sand flies, respectively. It is valuable to compare Figure 6 to the underlying mortality data that would traditionally be presented in tabular form (Table 2) and require much more internal conceptualization, with commensurate increased potential for error, by researcher and reader alike. The electronic map, if supplemented with meteorological data and a satellite photo base coverage, also facilitates rapid assessment of potential effects of the habitat on the pesticide applications. It should be noted that the inset wind rose diagram in Figure 7 has a wind angle that perfectly matches the angle of spatial mortality in the west grid, indicating that the spray truck should have started further to the east so that pesticide would have an opportunity to reach all sentinel cages. If mortality data is only been considered in tabular form, it may be easy to miss this weakness in the experimental design and assign a lower overall efficacy to that pesticide application equipment, biased by zero mortality values from areas not contacted by the pesticide.

In our experience, the majority of pesticide applications in the field produce a gradient of mortality through the target area sentinel cages, which automatically demonstrates that the application was valid. However, if zero mortality is observed throughout the treatment area and the spray event is valid (i.e., the spray cloud moving through the target sentinel area is observed), it can be inferred that the pesticide is not effective with that species at the rate applied, in that environment, and with that application equipment. Of course, this is given that the pesticide batch is not expired nor has been stored inappropriately. On the other hand, some aerial pesticide applications in particular may produce no visible or detectable spray cloud impacting the target area, and zero mortality throughout sentinels may mean that the spray missed the target area. It is advised to anticipate this scenario by setting up a series of extra sentinel cages that brackets the target impact area to some distance upwind and downwind (e.g., $50 \mathrm{ft}$ intervals for at least one swath width in each direction) so that some indication of touchdown of the pesticide may be gleaned if the target area is missed.

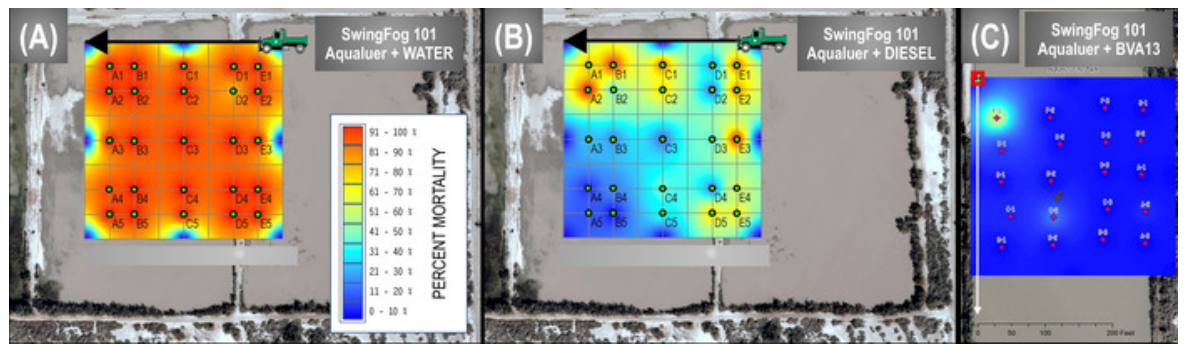

Figure 6: Representative interpolated sentinel cage mortality data from a field trial in hot-arid desert conditions targeting mosquitoes. In this series of sprays, the thermal fog pesticide sprayer, pesticide, and environment were kept constant, varying among pesticide diluent to (A) water, (B) diesel, and (C) BVA 13 mineral oil across the three trials. Please click here to view a larger version of this figure.

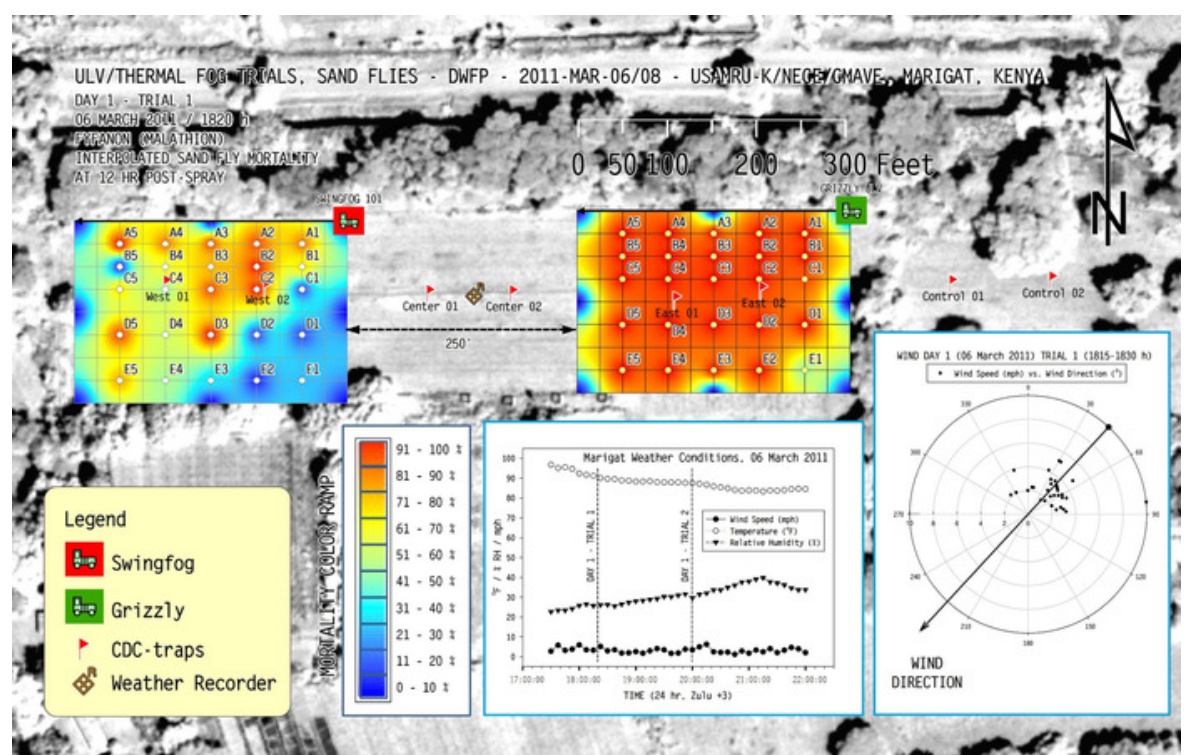

Figure 7: Representative interpolated sentinel cage mortality data from a field trial in hot-equatorial conditions targeting sand flies. In this set of two simultaneous spray applications the pesticide and environment were kept constant, but the pesticide sprayer was different between the two grids: thermal fog (west grid) and ultra-low volume (ULV, east grid). Note that the wind direction indicated in the wind rose diagram perfectly matches the angle of spatial mortality in the west grid, indicating that the spray truck should have started further to the east so that pesticide would have an opportunity to reach all sentinel cages. Please click here to view a larger version of this figure. 


\begin{tabular}{|c|c|c|c|c|c|c|}
\hline & \multicolumn{6}{|l|}{ PROJECT: } \\
\hline & \multicolumn{6}{|l|}{$\begin{array}{l}\text { ECOLOGICAL } \\
\text { ZONE: }\end{array}$} \\
\hline & \multicolumn{6}{|l|}{ LOCATION: } \\
\hline & \multicolumn{6}{|l|}{ FIELD SITE: } \\
\hline & \multicolumn{6}{|l|}{$\begin{array}{l}\text { DATE OF } \\
\text { APPLICATION: }\end{array}$} \\
\hline & \multicolumn{6}{|l|}{$\begin{array}{l}\text { SPRAY } \\
\text { EQUIPMENT: }\end{array}$} \\
\hline & \multicolumn{6}{|l|}{$\begin{array}{l}\text { PESTICIDE } \\
\text { FORMULATION: }\end{array}$} \\
\hline & \multicolumn{6}{|l|}{ DILUENT: } \\
\hline & \multicolumn{6}{|l|}{\begin{tabular}{l|} 
SENTINEL \\
INSECT SPECIES:
\end{tabular}} \\
\hline & \multicolumn{6}{|l|}{$\begin{array}{l}\text { LIFE STAGE OF } \\
\text { SENTINELS: }\end{array}$} \\
\hline & & \multicolumn{5}{|c|}{ MORTALITY CHECKS } \\
\hline & & \multicolumn{2}{|c|}{$\begin{array}{l}\text { [copied directly from sentinel cages; } \\
\text { data observed in field] }\end{array}$} & \multicolumn{3}{|c|}{ [observed from sentinel cages stored in trays] } \\
\hline & $\begin{array}{l}\text { Sentinel Cage } \\
\text { Code }\end{array}$ & No. PRE-DEAD & $\begin{array}{l}\text { No. DEAD POST- } \\
\text { SPRAY/HOLD } \\
\text { TIME }\end{array}$ & $\begin{array}{l}\text { No. DEAD } 6 \mathrm{hr} \\
\text { POST-SPRAY }\end{array}$ & $\begin{array}{l}\text { No. DEAD } 12 \mathrm{hr} \\
\text { POST-SPRAY }\end{array}$ & $\begin{array}{l}\text { No. DEAD } 24 \mathrm{hr} \\
\text { POST-SPRAY }\end{array}$ \\
\hline \multirow{25}{*}{ Treatment Area } & A1 & & & & & \\
\hline & \multicolumn{6}{|l|}{ A2 } \\
\hline & \multicolumn{6}{|l|}{ A3 } \\
\hline & \multicolumn{6}{|l|}{ A4 } \\
\hline & \multicolumn{6}{|l|}{ A5 } \\
\hline & \multicolumn{6}{|l|}{ B1 } \\
\hline & \multicolumn{6}{|l|}{ B2 } \\
\hline & \multicolumn{6}{|l|}{ B3 } \\
\hline & \multicolumn{6}{|l|}{ B4 } \\
\hline & \multicolumn{6}{|l|}{ B5 } \\
\hline & \multicolumn{6}{|l|}{$\overline{C 1}$} \\
\hline & \multicolumn{6}{|l|}{$\mathrm{C} 2$} \\
\hline & \multicolumn{6}{|l|}{ C3 } \\
\hline & $\mathrm{C} 4$ & & & & & \\
\hline & C5 & & & & & \\
\hline & D1 & & & & & \\
\hline & $\mathrm{D} 2$ & & & & & \\
\hline & D3 & & & & & \\
\hline & D4 & & & & & \\
\hline & D5 & & & & & \\
\hline & E1 & & & & & \\
\hline & E2 & & & & & \\
\hline & E3 & & & & & \\
\hline & E4 & & & & & \\
\hline & E5 & & & & & \\
\hline Control Area & Control 1 & & & & & \\
\hline
\end{tabular}




\begin{tabular}{|l|l|l|l|l|l|}
\hline Control 2 & & & & & \\
\hline Control 3 & & & & & \\
\hline Control 4 & & & & & \\
\hline Control 5 & & & & & \\
\hline Control 6 & & & & & \\
\hline Control 7 & & & & & \\
\hline Control 8 & & & & & \\
\hline Control 9 & & & & & \\
\hline Control 10 & & & & & \\
\hline
\end{tabular}

Table 1: Sample mortality data form. The form has spaces for general information about the field trial in the top left, which is critical to manage data from multiple field projects. The main section of the form has spaces for pre- and post-spray mortality (both copied directly from the sentinel cages), through the later $6 \mathrm{~h}, 12 \mathrm{~h}$, and $24 \mathrm{~h}$ checks. Handwritten data on this form are entered into a similar electronic spreadsheet, with added columns to correct mortality data for pre-spray dead and to correct spray area mortality for any environmentally-induced mortality in the control area.

\begin{tabular}{|c|c|c|c|}
\hline \multirow[b]{2}{*}{ Sentinel Cage Code } & \multicolumn{3}{|c|}{ PERCENT MORTALITY (ABBOTT-CORRECTED) } \\
\hline & (A) Aqualuer + Water & (B) Aqualuer + Diesel & (C) Aqualuer + BVA13 \\
\hline A1 & 1 & 0.78 & 0.54 \\
\hline A2 & 1 & 1 & 0.05 \\
\hline A3 & 1 & 0.29 & 0 \\
\hline A4 & 1 & 0.04 & 0.03 \\
\hline A5 & 1 & 0.04 & 0 \\
\hline B1 & 1 & 0.79 & 0 \\
\hline B2 & 1 & 0.82 & 0 \\
\hline B3 & 1 & 0.15 & 0 \\
\hline B4 & 1 & 0.21 & 0.15 \\
\hline B5 & 1 & 0 & 0 \\
\hline C1 & 1 & 0.93 & 0 \\
\hline $\mathrm{C} 2$ & 1 & 0.71 & 0.01 \\
\hline C3 & 1 & 0.32 & 0 \\
\hline C4 & 1 & 0.26 & 0 \\
\hline $\mathrm{C5}$ & 1 & 0.46 & 0 \\
\hline D1 & 1 & 0.67 & 0 \\
\hline D2 & 0.78 & 0.24 & 0.01 \\
\hline D3 & 0.97 & 0.5 & 0 \\
\hline D4 & 1 & 0.27 & 0.03 \\
\hline D5 & 1 & 0.33 & 0 \\
\hline E1 & 1 & 0.82 & - \\
\hline E2 & 1 & 0.88 & - \\
\hline E3 & 1 & 1 & - \\
\hline E4 & 1 & 0.79 & - \\
\hline E5 & 1 & 0.78 & - \\
\hline
\end{tabular}

Table 2: Underlying mortality data used to create the interpolated efficacy map in Figure 6. It should be noted that some spatial information can be captured in this table; for instance, this can be done by separating rows and columns by distance from sprayer and distance along spray line, respectively. However, temporal changes in mortality corresponding to immediate post-spray, $4 \mathrm{~h}$ post-spray, and $12 \mathrm{~h}$ post-spray, for example, would require a more complex table or additional tables. Similarly, separate tables for each diluent used in the trials are necessary for clarity if the tables are partitioned by space and time. 


\section{Discussion}

Combining the classic sentinel cage approach with electronic mapping of interpolated mortality data is a unique and powerful method to evaluate pesticides in the field, and it supports comparative pesticide efficacy studies across multiple environments and diverse configurations of pesticides and application techniques. Although the basic sentinel cage method is not new, the visualization of sentinel cage mortality patterns in a GIS is an advancement conducive to deeper analysis of patterns of flow of aerosol pesticide sprays. The interpolation of point measurements of pesticide efficacy into a color-coded map coverage is similar to adding smoke effusions to a wind tunnel to visualize air flow around an automobile, and it is a major improvement for reporting spatial and temporal mortality data in a series of tables.

Some parts of pesticide sprays can be seen with the naked eye, and pesticide droplets from invisible portions of sprays can be captured on glass slides or other media which, like sentinel cages, are long-established protocols. Evaporative products from droplets and droplets themselves can be captured with cotton ribbons and analyzed with a gas chromatograph/mass spectrometer, which provides even more information about the fate of a pesticide spray. However, the realized efficacy of the spray and spatial patterns of actual pesticide-induced mortality in the target area (which may include both droplet and evaporative product components) can only be definitively measured by sentinel insects.

Furthermore, the temporal component of efficacy can only be definitively measured by a series of mortality observations in sentinel insects that capture quantified indices of rapid knockdown versus long-term morbidity and mortality on the target species. Again, a series of interpolated color-coded maps can be used to clearly visualize the evolution of post-spray mortality over time, with an explicit spatial component, in a way that a series of tables is not able to communicate to a reader. The series of maps can be animated in a loop to reproduce the progress of the spray and its effects on sentinel insects, further enhancing the understanding of efficacy not only for a single trial, but in comparisons among pesticides, techniques, application equipment, target insects, and ecological zones.

For the highest quality mortality data in this method, great care should be taken throughout the start-to-finish handling and observations of the sentinel insects. Exposure time and conditions of sentinel insects to the environment across both the treatment and control areas before the pesticide spray should be as equal as practical. This exposure should include a period of acclimation to the ambient conditions and a uniform hold time post-spray for both treatment and control insects. Observations of mortality before the spray, after the hold time (i.e., during retrieval of cages), and for the designated periods post-spray should be carefully tracked on data forms. Care should also be taken to retrieve all sentinels from the field before departing for the lab. Control and treatment sentinel cages should be physically separated throughout the protocol. Reliable observations of background baseline mortality from the control cages at all designated time periods are critical to appropriate correction of observed mortality in the treatment zone. Accurate, precise, comparable, and meaningful efficacy maps can only be gained from high-quality mortality data input to the geographic information system.

The sentinel insect method is naturally flexible to be relevant in a variety of scenarios - anywhere a small cage can be placed, mortality data can be collected. For example, we have conducted pesticide trials with sentinel cages placed in and around simulated urban and rural buildings ${ }^{10}$ and U.S. military tents (unpublished data 2017-2018), in addition to multiple scenarios of desert, temperate, and tropical vegetation ${ }^{19,20,21,22}$ [including hoisting cages up to $60 \mathrm{ft}$ into pine canopy to measure vertical mortality following a large aerial application (unpublished data 2011-2017)]. If the ground is too hard to place sentinel cage poles or it is preferred to place them on concrete or asphalt areas, simple stands or concrete blocks can be constructed to support the poles. For scenarios to investigate sprayed liquid larvicides, the protocol can be modified to place empty plastic disposable 1 qt cups to capture larvicide across sentinel locations. These cups can be later filled with water and mosquito larvae to measure efficacy of the application ${ }^{7,23,24,25}$. Use floor tiles with the sticky side up to keep cups in place in wind and keep lids nearby to rapidly cap and collect following the post-spray hold time. Alternatively, cups can be left in place to weather naturally or left open in a controlled environment to investigate the longevity of a residual larvicide treatment.

To investigate droplet density and droplet spectra throughout the application area, slide spinners can be placed near sentinel insect positions - though exercise caution that the vortex from the spinning slides does not affect the flow of the pesticide spray to the sentinel insects. Similar to mapping mortality, additional columns in the attribute table for the sentinel locations can be added for droplet and dye parameters to derive interpolated coverages. Note that adding droplet collection aspects will demand an increase in the number of personnel in the field, with dedicated teams for example to carefully collect slides and assist the spray operator with dye additives. With additional materials and teams of personnel, these methods may be merged to conduct simultaneous trials using larval and adult sentinels, multiple application modes (aerial, ground, portable), or pesticides side-by-side (see representative results).

Although the main protocol was written for mosquitoes, we have successfully conducted field trials with sand flies and filth-breeding flies as sentinels with only minor modifications to the sentinel cages and overall protocol. For example, it is not practical to sex adult sand flies or filth breeding flies so that mixed-sex batches are used in sentinel cages, as this will reduce load on the colony because fewer specimens are needed than when working with mosquitoes. For sand flies, a very fine mesh must be used for the sentinel cages; furthermore, sand flies are not anaesthetized but instead added with aspirators directly into fully assembled cages through a rubber slit glued over a hole cut in the side of the cylinder. 


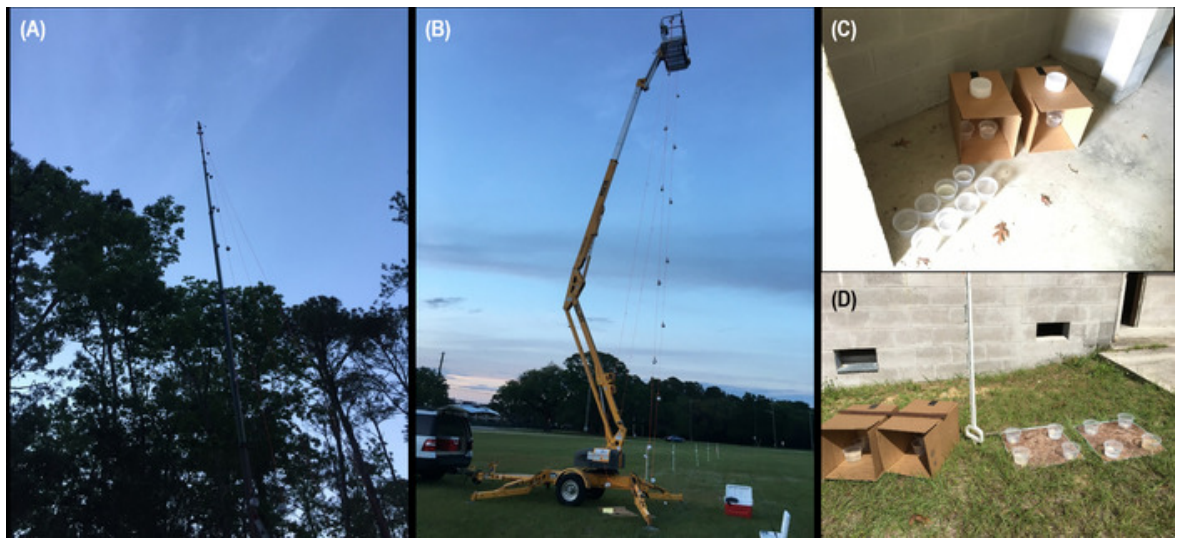

Figure 8: Additional scenarios demonstrating the flexibility of the sentinel system. The sentinel protocol for investigating pesticide efficacy in the field is very flexible, as shown in sentinel cages hoisted at intervals up to $60 \mathrm{ft}$ through pine canopy (A) and a nearby open area (B) to investigate capability of aerial ULV pesticide spray to penetrate canopy. The sentinel system can easily be adjusted to examine larvicide sprays targeting immature mosquito stages using disposable plastic cups to capture droplets (C) indoors and (D) outdoors in a simulated urban area. Please click here to view a larger version of this figure.

The efficacy mapping method is naturally flexible because it is based on interpolation which is a standard process in most GIS programs ${ }^{26}$. Generally, interpolation uses known data at set points to estimate data at nearby unsampled points. There exist several types of interpolation techniques ${ }^{27}$ which may be selected based on the spatial spread and density of the point mortality data. We have used inverse distance weighting (IDW), which assigns higher statistical weight to known data from points closer to the unknown points being estimated.

Troubleshooting for the field portion of the method is centered on control sentinel mortality (i.e., if mortality is $>25 \%$ in controls, and there is certainty that the application did not impact the control area, something in the environment other than the pesticide is causing mortality, which will confound analysis; then, the trial will need to be repeated or moved to another location). The most common vulnerabilities in the mapping portion of the method are production of quality data tables in the GIS, so it is critical to carefully paste data so that the right data are aligned with the right points (reading frame) and correctly label columns for each mortality time period, pesticide, application equipment, etc.

The sentinel insect method is not designed to be an absolute measure of efficacy. Rather, the method provides the ability to compare relative efficacy of a pesticide (under a given environment, application equipment, diluent, target insect, and technique) to the same pesticide under different conditions, or to compare different pesticides under the same conditions. The method does not include pesticide droplet or active ingredient capture, though apparatuses for investigating these aspects can easily be placed in the grid adjacent to sentinel cages. The sentinel cage method does not measure efficacy of pesticide sprays against vector insects in flight, which is possible but not practical ${ }^{28}$. A lively controversy exists whether the kind of mesh on sentinel cages effects measurements of pesticide spray efficacy ${ }^{14,29,30,31}$. However, this is not hugely relevant to our objective of investigating relative efficacy of a formulation across environments or several formulations within an environment (which can be conducted using a standard cage with a standard mesh type).

For example, a recent study compared sentinel cage mortality results across three similar aerial pesticide application experiments separated by decades, each using different sentinel cage methods, all with comparable results ${ }^{10}$. Similarly, controversy exists over whether to transfer sentinel insects to "clean" cages (i.e., cages that have not been sprayed). Again, data from sentinel insect mortality should be considered relative and not absolute and are not concerned with additional mortality resulting from sentinel insects contacting pesticide adhering to the cage or mesh. In fact, in natural environments, pesticide sprays will also adhere to natural surfaces that target insects may contact. We have previously found that mortality induced from handling mosquitoes, to include $\mathrm{CO}_{2}$ - or cold-based anaesthetization, may exceed mortality from contacting a pesticide that could have been present on cages (unpublished data 2008). Another limitation is that the relevance and applicability of sentinel cage mortality results in the local natural populations of the target insect still not being fully known; however, the closer genealogically the colonyreared sentinel insects are to the local species, the stronger the applicability of the efficacy maps is to local populations.

In future variations of this method, it would be beneficial to include modifications to accommodate pesticide applications with unmanned aerial systems (UAS). Current developments with UAS use in operational vector control include pesticide applications (in particular, larvicide formulations targeted at immature mosquito habitat) over and through highly inaccessible locations. To gain relevant information from sentinel trials, sentinel insect stations would need to be placed throughout inaccessible locations to the greatest extent possible. An example of relevant information is testing the capability of UAS effectively reaching a certain area with larvicide, with the only line-of-sight piloting by an operator who cannot directly observe the target area. This scenario may require development of other UAS to deploy and retrieve sentinel cages or larvicide collection cups and others to record meteorology in these inaccessible target areas. Mortality data from such a scenario can be analyzed in the GIS as with established scenarios, with added map features such as effects of natural obstacles, microhabitat, and micrometeorology that may differ from such effects during more standard applications with truck, aircraft, or portable sprayers. Advanced capabilities of GIS such as visualization of 3-dimensional interpolations of mortality from enhanced sentinel placement in vertical and horizontal grids are also possibilities for both standard and emerging application technology.

Disclosures

The authors have no conflicts of interest to disclose. 


\section{Acknowledgments}

We would like to thank the scientists and technicians at the Coachella Valley Mosquito and Vector Control District and the U.S. Army Medical Research Directorate-Kenya for expert production of colony insect specimens and collaboration on the field studies producing the unpublished data presented in the representative results. This research was supported by the U.S. Department of Agriculture (USDA)-Agricultural Research Service and the U.S. Department of Defense (DoD) Deployed War-Fighter Protection Program (DWFP). Mention of trade names or commercial products in this publication is solely for the purpose of providing specific information and does not imply recommendation or endorsement by the USDA, DoD, or DWFP. The USDA is an equal opportunity provider and employer.

\section{References}

1. Britch, S.C., et al. A mobile App for military operational entomology pesticide applications. Journal of the American Mosquito Control Association. 30 (3), 234-238 (2014).

2. Britch, S.C., et al. Evaluation of barrier treatments on native vegetation in a southern California desert habitat. Journal of the American Mosquito Control Association. 25 (2), 184-193 (2009).

3. Britch, S.C., et al. Residual mosquito barrier treatments on U.S. military camouflage netting in a southern California desert environment. Military Medicine. 175 (8), 599-606 (2010).

4. Britch, S.C., et al. Longevity and efficacy of bifenthrin treatment on desert-pattern US military camouflage netting against mosquitoes in a hotarid environment. Journal of the American Mosquito Control Association. 27 (3), 272-279 (2011).

5. Rogers, A.J., Beidler, E.J., Rathburn, C.J., Jr. A cage test for evaluating mosquito adulticides under field conditions. Mosquito News. 17 (3), 194-198 (1957).

6. Rathburn, Jr., C. B., Rogers, A.J., Boike, Jr., A, H., Lee, R.M. Evaluation of the ultra-low volume aerial spray technique by use of caged adult mosquitoes. Mosquito News. 29, 376-381 (1969).

7. Aldridge, R.L., Golden, F.V., Britch, S.C., Blersch, J., Linthicum, K.J. Truck mounted Natular 2EC (spinosad) ULV residual treatment in a simulated urban environment to control Aedes aegypti and Aedes albopictus in North Florida. Journal of the American Mosquito Control Association. 34 (1), 53-57 (2018).

8. Chaskopoulou, A., Latham, M.D., Pereira, R.M., Koehler, P.G. Droplet sampling of an oil-based and two water-based antievaporant ultra-low volume insecticide formulations using Teflon- and magnesium oxide-coated slides. Journal of the American Mosquito Control Association. 29 (2), 173-176 (2013).

9. Bonds, J.A.S., Greer, M.J., Fritz, B.K., Hoffmann, W.C. Aerosol sampling: comparison of two rotating impactors for field droplet sizing and volumetric measurements. Journal of the American Mosquito Control Association. 25 (4), 474-479 (2009).

10. Britch, S.C., et al. Aerial ULV control of Aedes aegypti with naled (Dibrom) inside simulated rural village and urban cryptic habitats. PLoS One. 13 (1), e0191555 (2018).

11. Knapp, F.W., Roberts, W.W. Low volume aerial application of technical malathion for adult mosquito control. Mosquito News. 25, 46-47 (1965).

12. Lofgren, C.S., Anthony, D.W., Mount, G.A. Size of aerosol droplets impinging on mosquitoes as determined with a scanning electron microscope. Journal of Economic Entomology. 66 (5), 1085-1088 (1973).

13. Haile, D.G., Mount, G.A., Pierce, N.W. Effect of droplet size of malathion aerosols on kill of caged adult mosquitos. Mosquito News. 42, 576-583 (1982).

14. Hoffmann, W.C., Fritz, B.K., Farooq, M., Cooperband, M.F. Effects of wind speed on aerosol spray penetration in adult mosquito bioassay cages. Journal of the American Mosquito Control Association. 24. (3), 419-426 (2008).

15. Aldridge, R.L., Wynn, W.W., Britch, S.C., Linthicum, K.J. Aspirator gun for high-throughput mosquito bioassays. Journal of the American Mosquito Control Association. 28 (1), 65-68 (2012).

16. Aldridge, R.L., et al. High-throughput mosquito and fly bioassay system for natural and artificial substrates treated with residual insecticides. Journal of the American Mosquito Control Association. 29 (1), 84-87 (2013).

17. Abbott, W.S. A method of computing the effectiveness of an insecticide. Journal of Economic Entomology. 18, $265-267$ (1925).

18. Healy, M.J.R. A table of Abbott's correction for natural mortality. The Annals of Applied Biology. 39 (2), $211-212$ (1952).

19. Britch, S.C., et al. Evaluation of ULV and thermal fog mosquito control applications in temperate and desert environments. Journal of the American Mosquito Control Association. 26 (2), 183-197 (2010).

20. Britch, S.C., et al. Evaluation of ULV applications against Old World sand fly (Diptera: Psychodidae) species in equatorial Kenya. Journal of Medical Entomology. 48 (6), 1145-1159 (2011).

21. Dunford, J.C., et al. SR450 and Superhawk XP applications of Bacillus thuringiensis israelensis against Culex quinquefasciatus. Journal of the American Mosquito Control Association. 30 (3), 191-198 (2014).

22. Haagsma, K.A., Breidenbaugh, M.S., Linthicum, K.J., Aldridge, R.L., Britch, S.C. Development of Air Force aerial spray night operations: high altitude swath characterizations. U.S. Army Medical Department Journal. 47-59 (2015).

23. Henke, J.A. Fighting Aedes aegypti in the desert. Proceedings and Papers of the Eighty-Fifth Annual Conference of the Mosquito and Vector Control Association of California. 85, 24-25 (2017).

24. Britch, S.C., et al. Ultra-low volume application of Natular 2EC (spinosad) larvicide as a residual in a tropical environment against Aedes and Anopheles species. Journal of the American Mosquito Control Association. 34 (1), 58-62 (2018).

25. Golden, F.V., et al. Ultra-low volume application of Natular 2EC (spinosad) as a residual in a hot-arid environment against Aedes aegypti. Journal of the American Mosquito Control Association. 34 (1), 63-66 (2018).

26. Hay, S.I., Lennon, J.J. Deriving meteorological variables across Africa for the study and control of vector-borne disease: a comparison of remote sensing and spatial interpolation of climate. Tropical Medicine \& International Health. 4, 58-71 (1999).

27. Tunalioğlu, N. Quality test of interpolation methods on steepness regions for the use in surface modelling. Technical Gazette (Tehnički vjesnik). 19 (3), 501-507 (2012).

28. Rathburn, C.B., Rogers, A.J. Field tests of mists and dusts against caged adults of Aedes taeniorhynchus (Wied.). Report of the Thirtieth Annual Meeting, Florida Anti-Mosquito Association. 38-43 (1959). 
29. Breeland, S.G. Effect of test cage materials on ULV malathion evaluations. Mosquito News. 30, 338-342 (1970).

30. Boobar, L.R., Dobson, S.E., Perich, M.J., Darby, W.M., Nelson, J.H. Effects of screen materials on droplet size frequency-distribution of aerosols entering sentinel mosquito exposure tubes. Medical and Veterinary Entomology. 2, 379-384 (1988).

31. Bunner, B.L., Posa, F.G., Dobson, S.E., Broski, F.H., Boobar, L.R. Aerosol penetration relative to sentinel cage configuration and orientation. Journal of the American Mosquito Control Association. 5 (4), 547-551 (1989). 